Relations industrielles

Industrial Relations

\title{
Cynthia A. Lengnick-Hall and Mark L. Lengnick-Hall. Interactive Human Resource Management and Strategic Planning
}

\section{Thierry Wils}

Volume 47, numéro 2, 1992

URI : https://id.erudit.org/iderudit/050782ar

DOI : https://doi.org/10.7202/050782ar

Aller au sommaire du numéro

Éditeur(s)

Département des relations industrielles de l'Université Laval

ISSN

0034-379X (imprimé)

1703-8138 (numérique)

Découvrir la revue

Citer ce compte rendu

Wils, T. (1992). Compte rendu de [Cynthia A. Lengnick-Hall and Mark L. Lengnick-Hall. Interactive Human Resource Management and Strategic Planning]. Relations industrielles / Industrial Relations, 47(2), 385-388. https://doi.org/10.7202/050782ar

Tous droits réservés (C) Département des relations industrielles de l'Université Laval, 1992
Ce document est protégé par la loi sur le droit d'auteur. L’utilisation des services d'Érudit (y compris la reproduction) est assujettie à sa politique d'utilisation que vous pouvez consulter en ligne.

https://apropos.erudit.org/fr/usagers/politique-dutilisation/ 
quelque peu de côté les effets de la négociation dans un contexte sectoriel ou multi-employeurs. Également, la comparaison des conditions du marché du travail fut difficile à cause d'une trop grande hétérogénéité des entreprises faisant partie de l'échantillon.

Même avec ces lacunes, ce modèle fait office de pionnier dans ce domaine et peut servir de balise à d'autres recherches.

Roch Laflamme

Université Laval

Interactive Human Resource Management and Strategic Planning, par Cynthia A. Lengnick-Hall et Mark L. Lengnick-Hall, New York, Quorum Books, 1990, 196 p., ISBN 0-89930-502-4

Une dizaine d'années se sont déjà écoulées depuis que l'idée d'aborder la gestion des ressources humaines selon une perspective stratégique a été proposée aux États-Unis (Dyer 1983). En dix ans, ce courant de recherche a réussi à attirer l'attention des chercheurs, comme en témoignent les nombreux écrits - parfois empiriques, parfois spéculatifs - sur le sujet (Butler, Ferris et Napier 1991). Le temps était donc venu de proposer une synthèse de ces travaux, ce qui s'est produit en France en 1988 (Besseyre des Horts), au Québec en 1991 (Wils, Le Louarn et Guérin) et aux États-Unis également en 1991 avec la parution du livre de Lengnick-Hall et Lengnick-Hall. Contrairement au livre américain de Fombrun, Tichy et Devanna (1984), ce livre n'est pas un recueil de textes mais une synthèse originale des écrits. La recension de cet ouvrage commencera par un résumé des différents chapitres afin de mettre en relief quelques interrogations que cette lecture nous a inspirées et se terminera par quelques commentaires plus généraux.

Le livre de Lengnick-Hall et Lengnick-Hall est composé de sept chapitres. Le premier chapitre intitulé Defining Strategic Human Resource Management ne propose pas vraiment une définition de la gestion stratégique des ressources humaines, mais explicite plutôt des postulats et des prescriptions. Par postulat, nous entendons des affirmations du genre "les ressources humaines sont une ressource critique pour bâtir un avantage compétitif". Si une telle affirmation était toujours vraie, on peut se demander pourquoi tant de dirigeants d'entreprise ne se servent pas davantage de cette ressource pour assurer leur survie. C'est alors que les auteurs ont recours à des prescriptions en précisant que cet avantage ne peut se concrétiser que dans la mesure où plusieurs prescriptions sont suivies. A titre d'exemple, une de leurs prescriptions se lit comme suit: Human resource management should be an integral part of the strategic planning processes of a firm (page 4). Somme toute, ce premier chapitre est une profession de foi en l'école du "choix stratégique" qui reconnait l'importance des décideurs. Le seul reproche qui pourrait leur être adressé est de ne pas avoir pris le temps de bien définir la gestion stratégique des ressources humaines et de proposer des postulats et des prescriptions sans en discuter leur bien-fondé.

Avant d'aborder le lien entre la ressource humaine et la gestion stratégique, il est logique de commencer par comprendre le concept de stratégie, 
ce que font les auteurs dans leur deuxième chapitre intitulé An Overview of Strategic Management. Ce chapitre présente en une vingtaine de pages un excellent survol du sujet. Les auteurs abordent successivement (a) la notion d'avantage compétitif par le biais de la typologie de stratégie de Porter (domination par les coûts, différenciation, etc.), (b) les concepts d'adaptation stratégique et de produit/marché à l'aide de la typologie de Miles et Snow (prospecteur, défenseur, etc.) et (c) un type d'analyse stratégique de portefeuille très connu, soit la grille du BCG (vache à lait, étoiles, etc.). Il est seulement dommage que les auteurs aient choisi de présenter la matière de façon séquentielle plutôt que synthétique et qu'ils aient passé sous silence les critiques dont fait l'objet actuellement la planification stratégique.

Dans le troisième chapitre, intitulé An Overview of Human Resource Management, les auteurs expliquent la transformation de l'administration du personnel en gestion des ressources humaines, symbolisant le passage de l'opérationnel au stratégique. Selon eux, les employés en tant que ressource doivent être gérés à l'aide des théories du capital humain ou de l'utilité. Leur argumentation à cet effet est peu convaincante. Jusqu'à présent la théorie de l'utilité n'a pas encore été appliquée en gestion stratégique des ressources humaines et risque de ne pas l'être avant longtemps parce que au niveau stratégique le qualitatif prime le quantitatif. Il aurait été sans doute plus intéressant d'expliquer pourquoi une telle transformation a eu lieu.

L'objet du quatrième chapitre intitulé Early Approaches to Strategic Human Resource Management est de discuter quatre écoles faisant soi-disant partie du mouvement de gestion stratégique des ressources humaines: la comptabilité des ressources humaines, la planification des ressources humaines, l'alignement des ressources humaines sur l'environnement et l'alignement sur la stratégie. L'argumentation de ce chapitre est très discutable. Premier point discutable, l'école de la comptabilité des ressources humaines qui a commencé au début des années 1970 n'appartient pas au courant stratégique qui, lui, a débuté au début des années 1980 (même s'il est vrai que des efforts de quantification en gestion des ressources humaines ont été déployés au début des années 1980). Un autre point discutable est la vision statique que les auteurs ont de la planification des ressources humaines. Pour eux, cette dernière tend à se réduire à la prévision des effectifs, ce qui correspond à une définition des années 1970. Depuis lors, le concept de planification des ressources humaines n'a cessé d'évoluer pour mieux intégrer, entre autres, l'aspect stratégique. Selon nous, les deux dernières écoles mentionnées par les auteurs ne peuvent être dissociées de la planification des ressources humaines. L'enjeu de la planification des ressources humaines est précisément d'aligner les ressources humaines non seulement sur l'environnement externe (la troisième école) et sur la stratégie (la quatrième école), mais également sur l'environnement interne (Wils, Le Louarn et Guérin 1991). En réalité, les différentes écoles doivent plutôt ètre considérées comme plusieurs couches de sédiments qui se sont déposées au fil des ans pour former la planification des ressources humaines telle que nous la connaissons aujourd'hui.

Le cinquième chapitre intitulé Critique of Old Assumptions and Articulation of New Assumptions se veut le prolongement du chapitre précédent. Étant donné nos réserves quant à leur interprétation des différentes écoles, nous ne pouvons 
être d'accord avec l'analyse critique qu'ils en font. A titre d'illustration, la planification des ressources humaines vue sous l'angle traditionnel de la prévision des effectifs est qualifiée de "réactive" parce qu'elle déduit la demande de personnel de la stratégie de l'organisation. Par contre, si la planification des ressources humaines est abordée sous l'angle contemporain, les considérations de ressources humaines sont analysées dès la formulation stratégique, ce qui la rend plus proactive. Le même commentaire s'applique quand les auteurs reprochent à la planification des ressources humaines de ne pas tenir compte des facteurs internes comme la culture.

Après avoir montré les soi-disant limites des différentes écoles, les auteurs proposent, dans le sixième chapitre intitulé The Proposed Approach, un modèle "proactif». Ils insistent à juste titre sur la nécessité d'une interaction simultanée entre les ressources humaines et la formulation stratégique. Pour ce faire, ils proposent une grille originale à deux dimensions: les attentes de croissance (corporate growth expectations) et le potentiel interne (organizational readiness). De cette grille émergent quatre situations stratégiques (expansion, development, productivity, redirection) qui sont par la suite abordées d'un point de vue de ressources humaines. Bien que la critique de cette grille dépasse le cadre de la présente recension, quelques commentaires sont néanmoins de mise. Premièrement, l'interaction proactive avancée par les auteurs ne constitue pas la seule façon d'intégrer la ressource humaine au processus stratégique (Wils, Le Louarn et Guérin 1991). Deuxièmement, on peut se demander si l'évaluation détaillée du potentiel interne a lieu lors de la formulation comme les autres prétendent ou bien, si elle se produit plus tard lors de l'étape de validation stratégique. Troisièmement, les auteurs suggèrent de suivre un processus adapté pour chacune des quatre situations stratégiques. Ne serait-il pas plus élégant de conceptualiser un processus général qui engloberait les quatre cas particuliers?

Dans le septième et dernier chapitre intitule Application of the Strategic Human Resource Management Model, les auteurs présentent une opérationnalisation de leur modèle sous la forme d'une série de questions (avec des échelles allant de 1 à 5). Cette partie du livre devrait intéresser les praticiens et les consultants. Cependant, force est de constater qu'il est partois plus tacile de poser les questions que d'y répondre. Par exemple, il est sans doute très subjectif de répondre à la question de la page 174 qui se lit comme suit: To what degree has minimal fit been achieved within this business unit?

En guise de conclusion, il nous semble important de soulever quelques questions de fond. Premièrement, les auteurs ne font pas de distinction entre analyse et décision. A titre d'exemple, dans le chapitre deux, ils prétendent s'intéresser aux décisions stratégiques, mais ils traitent de la grille du BCG qui est un outil d'analyse stratégique. Ou encore dans le chapitre six, ils proposent une grille qu'ils qualifient d'analytic approach alors qu'en fait il s'agit d'un processus de planification composé d'analyses et de décisions. De cette ambiguité en nait une autre, celle de la distinction entre planification et gestion. Le titre même du livre prête à confusion puisque gestion des ressources humaines et planification stratégique sont mises côte à côte. Cela veut-il dire que la gestion des ressources humaines est à ce point intégrée (voire absorbée) à la planification stratégique qu'il n'est plus nécessaire de parler de planification 
des ressources humaines? Une telle vision irait à l'encontre de la réalité puisque plusieurs études de cas ont montré l'interaction entre la planification stratégique et la planification des ressources humaines (Dyer 1984). Un autre aspect passé sous silence concerne les rôles que les professionnels de ressources humaines sont appelés à jouer dans ce processus de planification. La deuxième question de fond a trait à la vision très utilitariste adoptée par les auteurs pour intégrer les ressources humaines à la planification stratégique. Les auteurs insistent à plusieurs reprises sur l'importance des profits, de l'approche coût/bénéfice et de l'utilité. En filigrane le message de ce livre est le suivant: les intérêts des employés doivent passer après les impératifs économiques. Par exemple, à la page 2, on peut lire: The human resource department's responsibility should be defined as the maximisation of corporate profits through the better management and use of people. Ne vaudrait-il pas mieux parler de productivité que de simple maximisation des profits? Dans le même ordre d'idée, les auteurs émettent indirectement des réserves sur la compétence des professionnels de ressources humaines en écrivant: Human resource executives should be experienced business people (page 3). Le danger de cette prescription est de ne tenir compte que des intérêts des actionnaires (profits) au détriment de ceux des employés. Selon nous, ce n'est pas en mettant en sourdine les considérations sociales que les organisations vont réussir à redéfinir les relations d'emploi axées sur une plus grande coopération tant prônée pour surmonter le défi de la compétitivité.

Malgré ce biais, le livre de Lengnick-Hall et Lengnick-Hall vaut la peine d'être lu. Il aidera les étudiants et praticiens en relations industrielles à réaliser l'urgence de renforcer leurs compétences en gestion stratégique. Quant aux autres lecteurs, ils trouveront dans ce livre une synthèse originale des écrits en gestion stratégique des ressources humaines.

Thierry WILS

Université du Québec à Hull

Les besoins de formation en management: principes et méthodes de diagnostic, Série Formation à la gestion, $n^{\circ} 27$, par Milan Kubr et Joseph Prokopenko, Genève, Bureau international du travail, 1991, 317 p., ISBN 92-2-206399-6

L'évaluation des besoins de formation constitue le point de départ logique au développement et à l'évaluation des programmes de formation. L'évaluation rigoureuse des besoins de formation fournit des informations sur le niveau de formation nécessaire, la nature du contenu de la formation et les groupes d'employés susceptibles de recevoir de la formation.

Les recherches publiées sur le sujet se concentrent à priori sur le développement de nouvelles techniques et méthodes pour accroitre la qualité de l'information à recueillir lors de l'évaluation des besoins de formation. L'ouvrage de Kubr et Prokopenko s'avère essentiellement un guide méthodologique qui décrit la démarche à suivre pour effectuer un diagnostic complet et articulé des besoins de formation au niveau du management. Le livre dresse également l'inventaire des instruments en usage. Les auteurs portent une attention 